separate worker, a positive reaction was seen when challenged to a washing up liquid containing triclosan (another chlorine based substance). Due to the negative nature of the majority of tests, we considered the possibility that the chlorine itself may not be the provoking factor, but that chlorine-releasing formulations have the potential to react with amines from urine or bodily fluids when they are being used to disinfect clinical areas.

We report a 48 year-old health-care assistant for the elderly exposed to Haztabs (a chlorine-based cleaner) at work who had clear evidence of occupational asthma from serial peak expiratory flow records (Oasys score 3.44, ABC score $40 \mathrm{~L} / \mathrm{min} / \mathrm{hr}$, 5 positive timepoints), but negative tests to Haztab exposure when mixed with cold water. Adding urine (5\%) to the Haztab mixture resulted in a dual immediate and late asthmatic reaction (Figure 1). She showed mild airway hyper-reactivity pre challenge which was not repeated post challenge due to a continued low FEV1 of $39 \%$ predicted.

Conclusions The SIC results suggested that chloramines were the cause of her asthma, similar to the agents causing occupational asthma in workers exposed to indoor swimming pool vapours/ mists.

\section{P124 AN INVESTIGATION INTO COMORBIDITY ACCUMULATION IN ASTHMA PATIENTS WITH SYSTEMIC STEROID EXPOSURE}

${ }^{1} \mathrm{LB}$ Barry, ${ }^{1} \mathrm{JS}$ Sweeney, ${ }^{2} \mathrm{C}$ O'Neill, ${ }^{1} \mathrm{CP}$ Patterson, ${ }^{3} \mathrm{DP}$ Price, ${ }^{1} \mathrm{LH}$ Heaney. ${ }^{1}$ Queens University Belfast, Belfast, UK; ${ }^{2}$ National University of Ireland, Galway, Ireland; ${ }^{3}$ University of Aberdeen, Aberdeen, UK

\subsection{6/thoraxjnl-2016-209333.267}

Introduction Much of the economic cost associated with severe asthma is related to the treatment of comorbidities, several of which arise from systemic steroid exposure related to asthma management. However these comorbidities may not progress equally across age-groups and genders following systemic steroid exposure.
Aim To examine the relationship between age-groups and gender in relation to prevalence of steroid induced comorbidity and the associated costs.

Methods Data for a cohort of 808 patients with severe asthma (SA) matched by age and sex with a cohort of 3,975 patients with a diagnosis of mild asthma and 2,412 non-asthma patients with a diagnosis of rhinitis were extracted from the Optimum Patient Care Research Database (OPCRD). Consultation data were used to identify comorbidities and conditional logistic regression analysis provided odds ratios (95\% CI's) for comorbidity risk between cohorts by age-group and gender. Prescription data for individual comorbidities was costed using Prescription Cost Analysis data from Northern Ireland and regressed upon cohort, age and gender.

Results Results presented below focus on a comparison between the non-asthma and severe asthma cohorts. Compared to older patients, younger patients in the SA cohort show significantly higher odds ratios relative to the non-asthma cohort for many comorbidities (Table 1). Apart from chronic kidney disease, hypercholesterolemia, osteoporosis and osteopenia, males with SA have higher odds ratios for all other comorbidities listed in Table 1 relative to non-asthma patients than females with SA. Upon examination of costs for individual comorbidities, males cost more than females for type II diabetes, hypertension, psychiatric disorders, while the opposite was observed for osteoporosis and osteopenia. Significant linear and non-linear increasing relationships were observed across age for costs related to type II diabetes, hypertension, hypercholesterolemia, dyspeptic disorders, cardiovascular disease, osteoporosis and osteopenia. Furthermore cost differentials for age and gender exhibited varying relationships across morbidities.

Conclusions The odds of having many steroid-induced comorbidities are higher among younger persons; differences between genders are also evident. While patients' with SA cost significantly more than patients without steroid exposure or with mild steroid exposure, the distribution of the cost across age and gender varies across comorbidities.

Abstract P124 Table 1 Summary of odds ratios for comparison of comorbidity risk between severe asthma and rhinitis cohorts

\begin{tabular}{|c|c|c|c|c|c|c|}
\hline & \multicolumn{6}{|c|}{ Severe Asthma Cohort vs Rhinitis (Non-Asthma) Cohort - Odds Ratio ( $95 \%$ Confidence Intervals) } \\
\hline & Gender & & Age-group & & & \\
\hline & Male & Female & $\leq 45 \mathrm{yr}$ & $46-60 \mathrm{yr}$ & $61-70 \mathrm{yr}$ & $>70 \mathrm{yr}$ \\
\hline Type 2 Diabetes & $1.0(0.6-1.6)$ & $2.7(1.8-4.1)^{* * *}$ & $4.2(1.1-18.0)^{* *}$ & $4.0(2.2-7.2)^{* * *}$ & $1.7(0.9-3.2)$ & $0.9(0.5-1.6)$ \\
\hline Obesity & $1.8(1.3-2.4)^{* * *}$ & $2.8(2.2-3.5)^{* * *}$ & $3.7(2.4-5.6)^{* * *}$ & $3.3(2.4-4.6)^{* * *}$ & $1.8(1.3-2.6)^{* * *}$ & $1.8(1.3-2.6)^{* * *}$ \\
\hline Osteoporosis † & $49.2(11.2-217)^{* * *}$ & $4.6(3.2-6.8)^{* * *}$ & $18.3(6.4-52.8)^{* * *}$ & & $6.2(3.0-12.6)^{* * *}$ & $5.2(4.0-6.9)^{* * *}$ \\
\hline Osteopenia† & $\ddagger$ & $4.5(2.8-7.3)^{* * *}$ & $50.4(12.0-210)^{* * *}$ & & $2.7(1.2-6.3)^{* * *}$ & $3.1(1.5-6.6)^{* * *}$ \\
\hline Hypertension & $1.9(1.4-2.6)^{* * *}$ & $1.7(1.3-2.2)^{* * *}$ & $4.1(1.9-8.9)^{* * *}$ & $2.4(1.6-2.4)^{* * *}$ & $1.7(1.2-2.5)^{* * *}$ & $1.2(0.9-1.7)$ \\
\hline Chronic Kidney Disease $\dagger$ & $3.9(2.4-6.4)^{* * *}$ & $1.9(1.3-2.7)^{* * *}$ & $7.7(3.6-16.8)^{* * *}$ & & $3.2(1.7-5.9)^{* * *}$ & $1.4(1.0-2.1)^{*}$ \\
\hline Dyspeptic Disorders & $5.5(4.0-7.6)^{* * *}$ & $7.8(5.9-10.3)^{* * *}$ & $9.4(5.8-15.2)^{* * *}$ & $9.8(6.5-14.7)^{* * *}$ & $6.2(3.9-9.8)^{* * *}$ & $4.0(3.3-4.8)^{* * *}$ \\
\hline Psychiatric Disorders & $1.6(1.2-2.2)^{* * *}$ & $2.0(1.6-2.6)^{\star * *}$ & $2.6(1.8-3.9)^{* * *}$ & $2.1(1.6-2.9)^{\star * *}$ & $1.8(1.2-2.5)^{* * *}$ & $1.3(0.9-2.0)$ \\
\hline Sleep Disorders & $1.2(0.4-3.4)$ & $3.5(1.9-6.7)^{* * *}$ & $12.9(1.6-103)^{* *}$ & $8.1(2.1-30.3)^{* * *}$ & $2.3(0.7-7.5)$ & $0.6(0.2-1.8)$ \\
\hline Hypercholesterolemia & $2.1(1.4-3.1)^{* * *}$ & $1.4(1.0-1.9)^{*}$ & $\ddagger$ & $2.2(1.3-3.8)^{* * *}$ & $1.7(1.1-2.8)^{* *}$ & $1.2(0.8-1.8)$ \\
\hline
\end{tabular}

